\title{
Crecimiento económico, el multiplicador de comercio exterior de Harrod y el super-multiplicador de Hicks: Perú 1990 TI - 2014 T II
}

Economic growth, foreign trade multiplier Harrod and Hicks super-multiplier: Peru 1990 QI - 2014 Q II

Jorge Manrique Cáceres ${ }^{1}$ y Dévora Román Cruz ${ }^{1}$

\section{RESUMEN}

El trabajo analiza la influencia de las exportaciones sobre el crecimiento del PBI, asimismo analiza su influencia indirecta sobre los otros componentes del gasto autónomo. Como una ampliación del modelo de Harrod y del Supermultiplicador de Hicks, se introduce la variable importaciones, con el propósito de comprobar que también es influenciada por el aumento de las exportaciones. El tratamiento de la data es econométrico, con la inclusión de modelos VAR y MCE. Los resultados obtenidos evidencian una influencia directa e indirecta de las exportaciones en el crecimiento económico, con lo que se confirma la hipótesis formulada.

Palabras clave: crecimiento económico; exportaciones; modelo de Harrod; super multiplicador de Hicks.

\begin{abstract}
This research analyze the contribution of exports to GDP growth, this paper also analyze the indirect impacts of exports on the other components of autonomous spending. In addition, the imports variable is introduced as an extension of Harrod model and Hicks' super-multiplier to explain the indirect impacts of exports on imports. The analysis is based on the estimation of vector autoregressions and error correction models. The results make evident the direct and indirect contributions of exports to GDP growth, this is consistent with the hypothesis of this paper.
\end{abstract}

Keywords: economic growth; exports; Harrod'model; Hicks'super-multiplier.

\section{ICHIKLLACHAW}

Kay uryaqa yarpachatsikun ikspurtasiyun PBI miraynin nishqanpaqmi, hinaman riman wakin kunpunintikuna imanawpam kikinkunalla qillayta ushayanqanpaqmi, Harrud atskayaynintawan Hickspa hatun kaq atskayaynintawan, tsay ikspurtasiyunkunapaq baryabli nishqanmi yaykun, imanawmi impurtasiyunpa atskayayninkuna mirayan. Kay yarquyqa ikunumitrikum, kayman yaykun VARwan MCE nishqankunam. Kay ushay yarquykunaqa ikspurtasiyunkunapa tinkuyninwan mana tinkuyninkunam, ikunumikupa wiñayninchaw, tsaywanmi iputisis nishqan alli yarqushqa.

Pushaq shimikuna: ikunumikupa wiñaynin; impurtasiyunkuna; Harrudpa laayan; Hickspa hatun kaq atskayaynin.

1 Universidad Nacional «Santiago Antúnez de Mayolo». Huaraz, Perú. 


\section{INTRODUCCIÓN}

La búsqueda de las causas que han impulsado o frenado el crecimiento de las distintas economías, así como regularidades en el mismo, ha sido objeto de atención para muchos estudiosos en los últimos años. Aun cuando se ha obtenido resultados diversos, en algunos casos contradictorios, se ha encontrado evidencias de relaciones económicas directas y positivas entre las exportaciones y el crecimiento económico (Chow, 1986; Mesa, 1994; Sentsho, 2000; Crespo-Cuaresma y Wöez, 2000 y YifuLin y YongJun, 2002, entre otros, citados por Manalich, 2007).

... La integración a los mercados internacionales ha sido un objetivo permanente de los países, desde los años 60. A partir de este periodo se han acentuado los estudios sobre el tema y el interés se ha centrado en conocer cuál ha sido la influencia de las exportaciones sobre el crecimiento económico en los países con economías de mercado emergente (EME) o en vías de desarrollo, con particular énfasis en los análisis de índole empírica, en apoyo a los hacedores de política y orientadores de estrategias, para reducir desequilibrios económicos y acelerar el crecimiento... (Manalich, 2007: 2)

En el Perú todavía carecemos de suficientes estudios y análisis sobre el papel de las exportaciones sobre el crecimiento del Producto Bruto Interno y sobre todo medir su efecto multiplicador sobre el gasto autónomo, compuesto por el consumo privado y el gasto del gobierno. En esencia, interesa responder a la siguiente pregunta:

¿De qué manera las exportaciones tienen un efecto directo sobre el crecimiento del producto bruto interno y cómo, de manera indirecta, las exportaciones actúan sobre el gasto autónomo y sobre las importaciones manteniendo su impulso multiplicador?

El propósito de este trabajo es participar en el debate teórico existente acerca de la posible relación entre las exportaciones y el crecimiento del Producto Bruto Interno y su influencia indirecta sobre gasto autónomo y las importaciones, y, en particular, probar empíricamente la validez de la hipótesis del crecimiento inducido por las exportaciones (CIE), así como realizar una breve revisión de la literatura empírica existente sobre el tema; ello permitirá conocer enfoques sobre qué metodología utilizar para realizar el análisis.

El crecimiento económico ha sido, desde hace mucho tiempo, motivo de preocupación y estudio. Economistas como Smith (1776), Malthus (1789) y David Ricardo (1817) se ocuparon de investigar la fuente del crecimiento. Los dos primeros enfatizaron en el papel de la tierra como fuente de crecimiento, Gaviria (2005). David Ricardo enriqueció el trabajo de Smith, pues aunque ambos veían el papel que el comercio exterior tenía en el crecimiento, los entendían de forma diferente. Smith se apoyaba en las exportaciones y Ricardo en las importaciones.

En el siglo XX, con la aparición de importantes industrias, la acumulación del capital y las nuevas tecnologías pasaron a ser la fuente dominante en el desarrollo económico, surgió el modelo neoclásico de crecimiento, en el cual la inversión ocupa un lugar relevante. Se destacan en este período las teorías de Keynes (1936), Harrod (1939) y Domar (1946).

Keynes $^{2}$ (1936) con su trabajo «La teoría general de la ocupación, el interés y el dinero» y, más adelante, los modelos de Harrod (1939) y Domar (1946), que ampliaron y dieron un enfoque dinámico a las ideas Keynesianas, marcaron el desarrollo posterior de la teoría del crecimiento económico.

\footnotetext{
2 Keynes tiene un enfoque macroeconómico y presenta un modelo de corto plazo, para economía cerrada, en la cual la economía está dirigida por la demanda (De la Rosa, 2006).
} 
Entre los primeros en estudiar el vínculo entre el crecimiento económico y las exportaciones se encuentran Kaldor (1975), Verdoon (1980) y Hicks (1950). Kaldor, analiza la experiencia de un grupo de países desarrollados y concluye que el crecimiento económico de los mismos se debe a un grupo de hechos estilizados (generalizaciones empíricas), los cuales se conocen como las «leyes de Kaldor», mediante las cuales se explica las diferencias en las tasas de incremento de las fases de crecimiento económico de un país. Estas leyes asocian el crecimiento de la economía con la expansión de la producción manufacturera.

En sus estudios, Kaldor (1975) comienza a defender la idea del crecimiento inducido por la demanda y en su modelo de crecimiento inducido por las exportaciones combina un «modelo de crecimiento liderado por la demanda» (DLG) con la condición de equilibrio de la balanza de pagos.

El fundamento teórico de las ideas de Kaldor es el supermultiplicador de Hicks³ (1950): en una economía abierta, el ingreso está determinado por el volumen de exportaciones, las cuales vienen a representar el componente autónomo de la demanda; o sea que, en el largo plazo ${ }^{4}$, la tasa de crecimiento del producto puede estar dirigida por el crecimiento de la demanda autónoma, De la Rosa (2006). El Supermultiplicador de Hicks refleja el efecto directo e indirecto que tiene el incremento de las exportaciones sobre el crecimiento del producto (Hicks, 19505; De la Rosa, 2006).

Para demostrar la importancia de la demanda como determinante del crecimiento, Thirlwall (2002) propone un sencillo modelo de crecimiento dirigido por las exportaciones. La importancia de las exportaciones como componente de la demanda agregada se debe a tres características:

En primer lugar, las exportaciones son el único componente de la demanda verdaderamente autónomo. La mayor parte del incremento del consumo y de la inversión dentro del país depende a su vez del incremento del producto, mientras que las exportaciones quedan determinadas desde fuera (Thirlwall, 2002).

Segundo, las exportaciones constituyen el único componente de la demanda que puede pagar los requerimientos de las importaciones. Thirlwall (2002) señala que, si bien es cierto que otros componentes de la demanda pueden dirigir el crecimiento (crecimiento dirigido por el consumo, crecimiento dirigido por la inversión o crecimiento dirigido por el gasto público), cada uno de estos componentes implica también un incremento en la demanda por importaciones. Sin las ganancias de las exportaciones que puedan pagar por el incremento en las importaciones producto de la expansión de la economía ocasionada por la expansión de la demanda, el crecimiento se verá restringido. Thirlwall (2002) afirma que las exportaciones son de gran importancia si el equilibrio en la cuenta corriente de la balanza de pagos es un requisito en el largo plazo.

Lo que esto significa es que las exportaciones no solo tienen un efecto directo sobre la demanda, sino también un efecto indirecto al permitir que los otros componentes de la demanda aumenten más rápido de lo que aumentarían de otra forma.

El tercer aspecto por el cual las exportaciones son importantes es que permiten el incremento de las importaciones. Al favorecer el incremento de las importaciones, la

\footnotetext{
3 Se le llama «Supermultiplicador» de Hicks al efecto combinado del crecimiento de las exportaciones y del otro gasto autónomo en el crecimiento del ingreso (Thirlwall, 1992, citado por De la Rosa, 2006).

4 El modelo Hicksiano se desarrolla en el contexto del largo plazo, a diferencia del modelo Keynesiano utilizado tradicionalmente para explicar el efecto que las fluctuaciones a corto plazo del ingreso y el empleo tienen sobre el crecimiento económico (McCombie y Thirlwall, 1994).

5 Basado en Thirlwall (1992, citado por De la Rosa, 2006).
} 
economía se vuelve más productiva, pues es posible obtener bienes de capital de mayor tecnología que no se producen domésticamente. Este es un argumento basado en el lado de la oferta a favor del crecimiento dirigido por las exportaciones.

Hasta hoy este debate continúa, y esta misma divergencia entre las posiciones existentes acerca de la relación exportaciones-crecimiento económico ha llevado a la aparición de numerosos estudios empíricos que intentan respaldar uno u otro punto de vista. En el presente estudio intentaremos probar la validez para el Perú, del crecimiento inducido por las exportaciones (Jiménez, 2010).

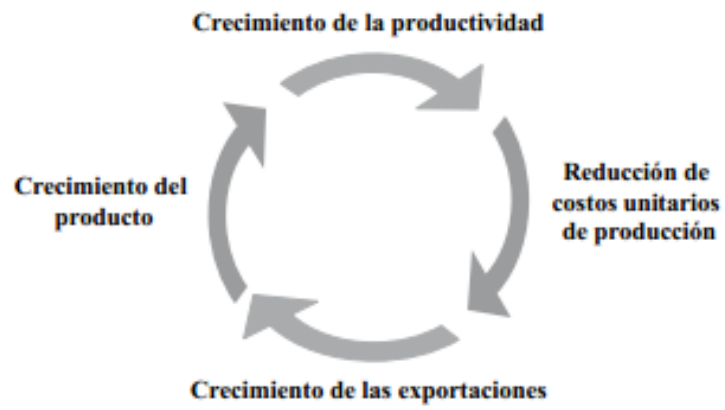

Figura 1. Thirlwall (2002: 56)

Hipótesis

Las exportaciones tienen un efecto directo sobre el crecimiento del producto bruto interno y, de manera indirecta, las exportaciones actúan sobre el gasto autónomo y las importaciones, manteniendo su impulso multiplicador.

Formulación del Modelo

$$
\begin{gathered}
\hline Y_{t}=\frac{X+E}{k} \mid Y_{t}=\left(X_{t}, E_{t}, M_{t}\right) \quad Y_{t}=\left(\frac{X}{k}\right)^{\alpha_{X}}\left(\frac{E}{k}\right)^{\alpha_{E}}\left(\frac{M}{k}\right)^{\alpha_{M}} \\
\dot{Y}_{t}=\alpha_{x}\left(\frac{\dot{X}_{t}}{k}\right)+\alpha_{E}\left(\frac{\dot{E}_{t}}{k}\right)+\alpha_{M}\left(\frac{M_{t}}{k}\right)+\mu_{t}
\end{gathered}
$$

Donde:

$\mathrm{Y}=$ producto bruto interno; $\mathrm{X}=$ exportaciones

$\mathrm{E}=$ gasto autónomo; $\mathrm{M}=$ importaciones

$\mathrm{k}=$ Multiplicador de comercio exterior de Harrod (a partir de la propensión marginal a impor$\operatorname{tar}) ; \alpha_{\mathrm{X}}, \alpha_{\mathrm{E}} \mathrm{y} \alpha_{\mathrm{M}}=$ son las proporciones de las exportaciones, del gasto autónomo y de las importaciones sobre el ingreso.

$\dot{Y}_{t}=$ Tasa de crecimiento del PIB; $\dot{X}_{t}=$ Tasa de crecimiento de las exportaciones; $\dot{E}_{t}=$ Tasa de crecimiento del gasto autónomo; $\dot{M}_{t}=$ Tasa de crecimiento de las importaciones; $\mu_{t}=$ Perturbación estocástica (considera todas las otras variables que influyen en el comportamiento del PIB).

\section{MATERIALES Y MÉTODOS}

En lo referente al tipo de investigación, esta es aplicada, descriptiva y correlacional, de diseño no experimental y longitudinal. La población en estudio está constituida por las variables macroeconómicas PIB, exportaciones, consumo privado, gasto del gobierno, importaciones, en el periodo 1990 TI - 2014 TII. Los datos usados son de frecuen- 
cia trimestral y abarcan el periodo comprendido desde 1990 al 2014 QII. Todas estas series están expresadas en valores corrientes y se transformaron a valores constantes, tomando como año base el 2007 y utilizando el deflactor implícito del PIB.

Esta información es tomada de fuentes secundarias, como el Instituto Nacional de Estadística e Informática, Banco Central de Reserva del Perú (BCRP). Se recopiló información estadística de reportes, memorias, boletines sobre las variables macroeconómicas en estudio. En el tratamiento metodológico se hace uso de la econometría de series temporales, el análisis de no-estacionariedad, técnicas de cointegración sobre modelos de vectores autorregresivos (VAR) y la modelización a través de mecanismos de corrección de error (MCE), analizando por ultimo las relaciones de causalidad de Granger y las funciones impulso-respuesta. Se usó el programa Eviews para el procesamiento de los datos.

\section{RESULTADOS}

Las series analizadas: producto bruto interno (PIB), exportaciones $(\mathrm{X})$, gasto autónomo (E) e importaciones (M), son de frecuencia trimestral, y abarcan el periodo comprendido desde 1990 Q1 hasta 2014 Q2. Asimismo, dichas series están expresadas en términos reales (a precios constantes de 2007); y, todas las series fueron desestacionalizadas. ${ }^{6}$

Tabla1. Cálculo del Multiplicador de Comercio Exterior de Harrod (k)

\begin{tabular}{ccccc}
\hline \multicolumn{4}{c}{ Dependent Variable: M } \\
Variable & Coefficient & Std. Error & t-Statistic & Prob. \\
\hline C & -7692.513 & 437.899 & -17.56685 & 0.0000 \\
PIB & 0.334421 & 0.00620 & 53.87904 & 0.0000 \\
R-squared & 0.967989 & Adjusted R-squared & 0.9676 \\
Durbin-Watson & 0.970272 & Prob (F-statistic) & 0.0000 \\
\hline
\end{tabular}

Partimos por el cálculo del multiplicador de comercio exterior de Harrod (k), definido como el inverso de la propensión marginal a importar. ${ }^{7}$ Los cálculos realizados sugieren que $k$ toma un valor de 2.990243. El multiplicador refleja el efecto directo e indirecto del crecimiento de las exportaciones sobre el crecimiento del producto.

Las variables exportaciones, gasto autónomo e importaciones se dividieron entre el multiplicador de Harrod, de acuerdo con el modelo especificado en el apartado anterior.

1. Análisis de estacionariedad de las series

En el periodo de estudio, se ha determinado que las variables analizadas son todas no estacionarias, es decir, son de orden I (1), pero son estacionarias en primeras diferencias I (0). Esto es resultado de la aplicación de la prueba Raíz Unitaria de Dickey-Fuller Aumentada.

\footnotetext{
${ }^{6}$ Usando el procedimiento Census X12.

$7 \mathrm{k}=1 / \beta$, donde $\beta$ es la propensión marginal a importar. $\beta=0.334421$.
} 
Tabla 2. Estacionariedad de series

\begin{tabular}{ccccccc}
\hline \multirow{2}{*}{$\begin{array}{c}\text { Variable } \\
(\text { en } \\
\text { logaritmos }\end{array}$} & Estadístico-t & Prob. & $\begin{array}{c}\text { Orden de } \\
\text { Integración }\end{array}$ & Estadístico-t & Prob. & Orden de \\
\cline { 2 - 6 } & \multicolumn{5}{c}{ Integración } \\
\hline En niveles* & \multicolumn{3}{c}{ En primeras diferencias } \\
\hline PIB & -3.046 & 0.126 & $\mathrm{I}(1)$ & -3.496 & 0.001 & $\mathrm{I}(0)$ \\
$\mathrm{X}$ & -1.343 & 0.871 & $\mathrm{I}(1)$ & -11.941 & 0 & $\mathrm{I}(0)$ \\
$\mathrm{E}$ & -1.753 & 0.719 & $\mathrm{I}(1)$ & -4.562 & 0 & $\mathrm{I}(0)$ \\
$\mathrm{M}$ & -2.54 & 0.309 & $\mathrm{I}(1)$ & -8.924 & 0 & $\mathrm{I}(0)$ \\
\hline
\end{tabular}

Contraste de Cointegración de Engle y Granger (MacKinnon, 1999)

Dado que las series PIB, X, E y M resultaron ser integradas de orden I(1), se procedió a analizar la presencia de cointegración entre dichas variables aplicando la metodología de Engle y Granger en dos etapas ${ }^{8}$, para lo cual partimos de la especificación y estimación de la siguiente relación de equilibrio a largo plazo.

$$
Y_{t}=\beta_{0}+\beta_{1}\left(\frac{X}{k}\right)_{t}+\beta_{2}\left(\frac{E}{k}\right)_{t}+\beta_{3}\left(\frac{M}{k}\right)_{t}+\mu_{t}
$$

Tabla 3. Contraste de Cointegración

\begin{tabular}{ccccc}
\hline \multicolumn{5}{c}{ Dependent Variable: PIB } \\
Variable & Coefficient & Std. Error & t-Statistic & Prob. \\
\hline $\mathrm{C}$ & 2.304370 & 0.535409 & 4.303941 & 0.0000 \\
$\mathrm{X} / \mathrm{k}$ & 0.090639 & 0.026571 & 3.411186 & 0.0010 \\
$\mathrm{E} / \mathrm{k}$ & 0.789234 & 0.053796 & 14.67093 & 0.0000 \\
$\mathrm{M} / \mathrm{k}$ & 0.030538 & 0.019940 & 1.531453 & 0.1091 \\
$\mathrm{~T}^{*}$ & 0.001628 & 0.000721 & 2.259738 & 0.0262 \\
R-squared & 0.997566 & Adjusted R-squared & 0.9974 \\
Durbin-Watson & 1.429907 & Prob(F-statistic) & 0.0000 \\
\hline
\end{tabular}

Los resultados completos de la estimación se presentan en el cuadro. La relación estimada a largo plazo queda establecida de la siguiente manera:

$$
\begin{gathered}
\widehat{Y}_{t}=2.3044+0.0906\left(\frac{X}{k}\right)_{t}+0.7892\left(\frac{E}{k}\right)_{t}+0.0305\left(\frac{M}{k}\right)_{t}+0.0016 t \\
R^{2}=0.9975 \quad D W=1.42
\end{gathered}
$$

Considerando los resultados de la regresión expuestos, se realizaron las pruebas de cointegración basándonos en los residuos con el fin de confirmar una posible relación de largo plazo entre las variables del modelo. La condición de cointegración de Engle y Granger, exige que los residuos de la regresión ${ }^{9}$ presenten un comportamiento estacionario.

La regresión realizada con las variables de estudio, usando como variable dependiente al PIB y como variables explicativas a las exportaciones, el gasto autónomo y las im-

\footnotetext{
8 La metodología de Engle y Granger en dos etapas consiste en construir los residuos a partir de una relación funcional especificada y aplicar tests de raíz unitaria a dichos residuos para determinar si existe cointegración (Primera Etapa). Si hay cointegración, se estima un Modelo de Corrección de Errores (Segunda Etapa).

9 Los residuos estimados de la regresión son: $\widehat{\mu}_{t}=Y_{t}-\beta_{0}-\beta_{1}\left(\frac{X}{k}\right)_{t}-\beta_{2}\left(\frac{E}{k}\right)_{t}-\beta_{3}\left(\frac{M}{k}\right)_{t}-\beta_{4} t$
} 
portaciones, arrojó una serie de errores que fueron estacionales, es decir, cuyo orden de integración fue cero.

Tabla 4. Residuos de la regresión

\begin{tabular}{cccc}
\hline \multirow{2}{*}{ Variable } & \multicolumn{3}{c}{ Dickey-Fuller aumentada en niveles, sin intercepto ni tendencia } \\
\cline { 2 - 4 } & Estadístico-t & Prob. & Orden de Integración \\
RESID 1 & -7.352 & 0.000 & $\mathrm{I}(0)$ \\
\hline
\end{tabular}

Por lo que se determinó que dichas variables estaban cointegradas.

Dado que las variables están cointegradas, entonces se puede utilizar los residuos de la regresión de equilibrio para estimar el MCE.

Modelo de Corrección de Errores (MCE), para ligar el comportamiento a corto plazo de las variables con el comportamiento a largo plazo de las mismas (Gujarati, 2010).

$$
\dot{Y}_{t}=\alpha_{1}\left(\frac{\ddot{y}}{k}\right)_{t}+\alpha_{2}\left(\frac{\ddot{E}}{k}\right)_{t}+\alpha_{3}\left(\frac{\ddot{y}}{k}\right)_{t}+\gamma\left[Y_{t-1}-\beta_{0}-\beta_{1}\left(\frac{X}{k}\right)_{t-1}-\beta_{2}\left(\frac{E}{k}\right)_{t-1}-\beta_{3}\left(\frac{M}{k}\right)_{t-1}\right]+v_{t}
$$

Donde: $\gamma$ es el coeficiente del mecanismo de corrección de errores.

Tabla 5. Correción de errores

\begin{tabular}{ccccc}
\hline \multicolumn{5}{c}{ Dependent Variable: DPIB } \\
Variable & Coefficient & Std. Error & t-Statistic & Prob. \\
\hline C & 0.003498 & 0.001719 & 2.0347 & 0.0448 \\
DX & 0.042010 & 0.028556 & 1.8711 & 0.0947 \\
DE & 0.596992 & 0.066614 & 8.9619 & 0.0000 \\
DM & 0.051850 & 0.022515 & 2.3028 & 0.0235 \\
RESID 1(-1) & -0.633055 & 0.098825 & -6.4058 & 0.0000 \\
R-squared & 0.581159 & Adjusted R-squared & 0.5629 \\
DW & 2.107207 & Prob(F-statistic) & 0.0000 \\
\hline
\end{tabular}

Cabe destacar que en este modelo se trabaja con la tasa de crecimiento de las variables, ya que la primera diferencia de los logaritmos de las variables es equivalente a la tasa de crecimiento de las mismas. Al estimar el MCE, se obtiene el siguiente resultado:

$$
\begin{gathered}
\hat{\dot{Y}}_{t}=0.0035+0.042\left(\frac{\ddot{y}}{k}\right)_{t}+0.5969\left(\frac{\ddot{y}}{k}\right)_{t}+0.0519\left(\frac{\ddot{y}}{k}\right)_{t}-0.6331 \hat{u}_{t-1} \\
R^{2}=0.5629 \quad D W=2.11
\end{gathered}
$$

El coeficiente - 0.63 es el mecanismo de corrección de errores, el cual presenta el signo correcto (negativo) y es significativo. Ello revela que aunque en el largo plazo hay una relación de equilibrio entre las variables del modelo de cointegración, en el corto plazo se producen desequilibrios. El signo negativo actúa para corregir el desequilibrio en el próximo periodo. 
2. Análisis de Causalidad

Para complementar el análisis se realizó la prueba de Causalidad de Granger ${ }^{10}$ para el grupo de variables de interés: PIB, X, E, M, con la finalidad de determinar si una variable precede a la otra, dicha prueba se realizó incluyendo 10 rezagos de las variables.

De acuerdo a los resultados obtenidos, se determinó que la tasa de crecimiento de las exportaciones 0 causa, en el sentido de Granger, a la tasa de crecimiento del PIB O, no siendo así en la dirección contraria, confirmándose la relación de causalidad unilateral de crecimiento de exportaciones a crecimiento del PIB.

Tabla 6. Relaciones de causalidad

\begin{tabular}{lccc}
\hline \multicolumn{3}{c}{ Pairwise Granger Causality Tests } & \\
Null Hypothesis: & Obs & F-Statistic & Prob \\
\hline DX does not Granger Cause DPIB & 87 & 2.37060 & 0.018 \\
DPIB does not Granger Cause DX & & 1.71516 & 0.095 \\
DE does not Granger Cause DPIB & 87 & 3.10109 & 0.002 \\
DPIB does not Granger Cause DE & & 4.78496 & 0.000 \\
DM does not Granger Cause DPIB & 87 & 1.99473 & 0.047 \\
DPIB does not Granger Cause DM & & 2.70560 & 0.007 \\
\hline
\end{tabular}

Del mismo modo se encontró que existe una relación de causalidad bilateral de crecimiento del gasto autónomo $(\dot{E})$ y crecimiento de las importaciones $(\dot{M})$ a crecimiento del PIB.

Funciones Impulso Respuesta ${ }^{11}$

Para obtener las funciones de impulso respuesta construimos el modelo VAR y derivamos las Funciones Impulso Respuesta.

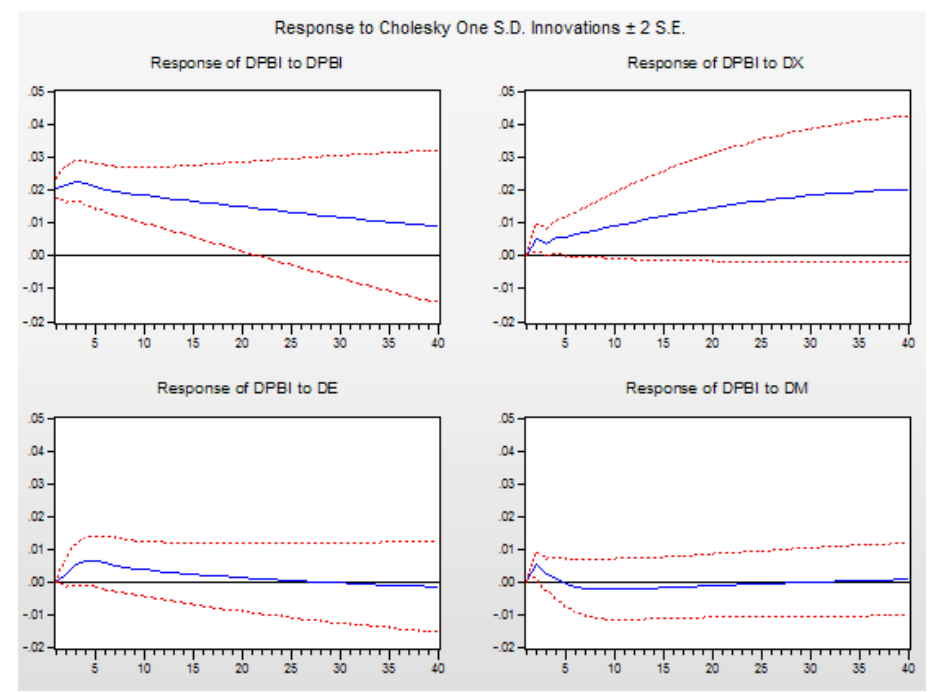

Figura 2. Funciones de impulso respuesta

\footnotetext{
${ }^{10}$ Consiste en comprobar que el comportamiento actual y pasado de una serie de tiempo «X» predice la conducta de una serie de tiempo «Y».

${ }^{11}$ Las funciones de impulso respuesta ilustran el efecto que tiene un shock aleatorio en una de las variables sobre el resto de las variables del sistema.
} 
Dado que el interés del presente trabajo recae sobre las variables Crecimiento Económico y Exportaciones, se presentan los gráficos de respuesta de la variable ante un impulso en las variables; asimismo se presenta la respuesta de las variables ante un impulso en la variable, siendo el horizonte empleado de 40 periodos.

Un shock positivo de las exportaciones produce un aumento progresivo del PIB en los periodos posteriores. Por su parte, un shock positivo del Gasto Autónomo sobre el PIB induce un efecto inicialmente positivo, el cual se amortigua hasta desaparecer a partir del periodo 20. De manera similar ocurre con un shock positivo de las importaciones sobre el PIB, el cual produce un efecto inicialmente positivo que se amortigua hasta desaparecer a partir del periodo 5 .

Un shock positivo de las exportaciones tiene un efecto positivo sobre el PIB, sobre el gasto autónomo y las importaciones.

\section{DISCUSIÓN}

Los resultados obtenidos en el estudio, apoyan la hipótesis teórica que sugiere que las exportaciones tienen un efecto positivo sobre el crecimiento económico, las funciones impulso-respuesta elaboradas muestran que dicho efecto es directo puesto que las exportaciones son un componente de la demanda agregada, e indirecto, puesto que las exportaciones inciden en los componentes del gasto autónomo, lo cual evidencia la magnitud del multiplicador de Hicks.

Del mismo modo, se aporta evidencia empírica a favor del modelo de crecimiento dirigido por las exportaciones (Thirlwall, 2002). Utilizando el análisis de cointegración y de causalidad se encontró que existe una influencia directa de las exportaciones (consideradas componente de la demanda verdaderamente autónomo) sobre el crecimiento económico; asimismo se evidencia que las exportaciones influyen sobre los componentes del gasto autónomo (consumo privado y gasto de gobierno), lo cual a su vez implica un incremento de las importaciones.

\section{CONCLUSIONES}

Los resultados obtenidos verifican la hipótesis de Crecimiento Inducido por las Exportaciones (CIE) para el caso peruano: el aumento de las exportaciones constituye uno de los factores que influye en el crecimiento económico.

Las exportaciones tienen influencia indirecta en el comportamiento del gasto autónomo (compuesto por el consumo privado (CP) y los gastos del gobierno (G), los cuales han sido agregados).

El comportamiento de las importaciones es inducido por el comportamiento de las exportaciones (a mayor exportación mayor disponibilidad de divisas, baja el tipo de cambio y consecuentemente crecen las importaciones).

El uso de técnicas de cointegración y el modelo de corrección de errores (MCE) para un periodo de 98 trimestres, comprendido entre 1990 y 2014 Q2 permitieron verificar la hipótesis planteada acerca de que las exportaciones tienen un efecto directo sobre el PIB. A su vez, las exportaciones explican no solo los cambios en el corto plazo, sino también el comportamiento del producto a largo plazo. El análisis de causalidad confirmó la hipótesis del CIE. 
$\mathrm{El}$ análisis de las funciones impulso respuesta muestra el efecto directo del crecimiento de las exportaciones sobre el crecimiento del PIB. Asimismo muestra que el crecimiento de las exportaciones tiene un efecto positivo en el crecimiento del gasto autónomo y el crecimiento de las importaciones, por lo que también se evidencia su efecto indirecto sobre el crecimiento del PIB, ya que actúa sobre el resto de la demanda manteniendo un impulso multiplicador.

\section{AGRADECIMIENTOS}

A los colegas del Doctorado en Economía de la UNMSM por sus valiosas sugerencias, en especial al $\mathrm{PhD}$. Manuel Uriarte por su paciencia en la revisión del presente trabajo.

\section{REFERENCIAS BIBLIOGRÁFICAS}

De La Rosa, Juan. 2006. Dos enfoques teóricos sobre el proceso de crecimiento económico: con énfasis en las exportaciones manufactureras. Análisis Económico. Vol. XXI, N 48, Tercer cuatrimestre.

Domar, Evsey. 1946. Capital Expansion, Rate of Growth and Employment. Econométrica, $14, \mathrm{~N}^{\circ} 2$.

Gaviria, Mario y Sierra, Hedmann. 2005. Lecturas sobre Crecimiento Económico Regional. <www.eumed.net/libros/2005/mgr/> [Consulta: 14-08-2014].

Gujarati, Damodar. 2010. Econometría. Quinta Edición. Mc GrawHill. México. D F.

Harrod, Roy. 1939. An Essay in Dynamic Theory. Economic Journal, The Economic Journal, 49, $\mathrm{N}^{\circ} 193$.

Hicks, John. 2010. A Contribution to the Theory of the Trade Cycle. Oxford University Press.

Jiménez, Félix. 2010. Crecimiento Económico: Enfoques y Modelos. Documento de Economía $\mathrm{N}^{\circ}$ 306. Lima: PUCP.

Kaldor, Nicholas. 1975. A Model of Economic Growth. The Economic Journal, 57; 591.

Keynes, Jhon. 1936. The General Theory of Employment, Interest, and Money. <http:// cas.umkc.edu/economics/people/facultypages/kregel/courses/econ645/ winter2011/generaltheory.pdf $>$ [Consulta: 05-01-2015].

MacKinnon, James. 1999. «Critical Values for Cointegration Tests». Cap. 13. En Long Run Economic Relationships: Readings in Cointegration, editado por R.F. Engle y V. W. J. Granger. Oxford: Oxford University Press.

Mañalich, Isis. 2007. Exportaciones y Crecimiento Económico. Evidencia empirica para Cuba. La Habana: Universidad de La Habana y Universidad de Oviedo.

Thirlwall, Anthony. 2002. The nature of Economic Growth: An alternative Framework for understanding the performance of nations. Cheltenham, UK.

Fecha de recepción: 22 de enero de 2015

Fecha de aceptación: 05 de abril de 2015

\section{Correspondencia}

Jorge Manrique Cáceres

j.manrique.c@hotmail.com 